\title{
Reform 2.0: Augmenting Innovative Mental Health Interventions
}

\author{
Leonard Bickman ${ }^{1,2}$
}

Published online: 12 January 2021

(c) The Author(s), under exclusive licence to Springer Science+Business Media, LLC part of Springer Nature 2021

\section{Introduction}

The September issue of this journal (Bickman 2020) included an extensive article that I authored that described the current state of mental health services, identified five critical problems, and suggested how to solve them. I focused on the potential contributions of artificial intelligence and precision mental health to improving mental health services. Based on reactions and conversations about that article I wanted to place my concerns in a broader context of reform of mental health services. I also wanted to describe a recent activity that I and some of my colleagues have undertaken to move that reform forward. I am using the editorial mechanism to publish this article. I take the privilege of publishing editorials seriously since they do not undergo peer review and are at my discretion as the editor. I have published fewer than a half dozen editorials in the over 20 years as the editor. This editorial focuses on services designed for children and families but I believe the discussion is also relevant to adult mental health services.

\section{Recognizing the Problem with Services as Usual}

There is extensive literature on the problems with what has been termed treatment as usual or care that is typically provided in community settings. The three major concerns I

Leonard Bickman wants to thank some of his colleagues at PRAC/ FRI for their input on an earlier draft of this editorial: Besa Bauta, Kim Hoagwood, Sylvia Rowlands, Tom Sexton and Lori Wheeless

Leonard Bickman

lbbickman@gmail.com

1 Feedback Research Institute, Boynton Beach, FL 33473, USA

2 Center for Children and Families; Psychology, Academic Health Center 1, Florida International University, 11200, Southwest 8th Street, Room 140, Miami, FL 33199, USA previously detailed include; lack of service accessibility, the inadequacy of implementation, and the limited effectiveness of these services. Much has been written these concerns. For example, Weisz et al. (2019), based on a review of 453 RCTs over 50 years, found that the mean effect size for treatment did not improve significantly for anxiety and ADHD and decreased significantly for depression and conduct problems. The authors concluded that there was a common pattern of treatment effects that were either unaffected by treatment or declined across the the 50 years for each of the target problems.

\section{Reform 1.0}

The field of mental health services did not acknowledge that a specific crisis existed in response to questions about our ability to deliver mental health services. However, contemporaneously several uncoordinated innovations occurred focusing primarily on improving effectiveness that I call Reform 1.0. These included:

- Continuous quality improvement (Bickman and Noser 1999; Chowanec 1994),

- Evidence-based treatments (APA, 2004; Chorpita et al. 2002),

- Measurement feedback systems_-MFSs (Bickman 2008; Lambert et al. 2003),

- Digital and mobile solutions (Hedlund, et al. 1985; Strecher 2007),

- Data-driven decision-making (Burns and Friedman 1990; Hedlund et al. 1985) and

- Systems of care and wraparound services (Bickman et al. 2003).

The above citations are meant to reflect the publications from the circa of reform 1.0 and not the most recent publications about these innovations.

All of these approaches have some theoretical support and/or evidence of efficacy under controlled conditions. 
However, their effectiveness significantly declined in realworld practice (Lyon et al. 2020). Moreover, these innovations experienced difficulty in being widely adopted. These approaches, especially the plethora of digital applications, suffer from low engagement and high dropout of both clients and professionals (Linardon and Fuller-Tyszkiewicz 2020). While there is a multitude of causes suggested for the lack of widespread adoption and diminished effectiveness, these approaches share common features that limit their success in actual clinical settings. It is critical to stress that I am not advocating abandoning these real advances in services, but instead believe that we can augment these innovations to optimize their adoption and effectiveness.

\section{What are the Problems with Reform 1.0?}

Reform 1.0 innovations include typical processes of collection of data, analyses of that data, and feedback of that data as information to decision-makers. For example, the success of evidence-based treatments depends upon implementing the treatment with fidelity, having criteria for successful implementation, an analytic approach to judging fidelity, and feedback to the clinicians to improve adherence to practice. Measurement feedback systems require the collection of data to assess client progress, an analytic approach to judge improvement, and feedback to the clinician about such progress. Decision support systems require the establishment of evidence or theory-based decision points and recommendations about the clinical decision based on data collected. The systems of care and wraparound models were distinguished in their early recognition of the importance of personalization or fitting the treatment to the individual. However, systems of care lacked a specific methodology to support personalization. Moreover, clinicians trained in the system of care approach did not agree on what level of care would be best for children (Bickman et al. 1997). However, as described below, Reform 2.0 offers these system level innovations the potential to accurately match clients with appropriate services.

In summary, these approaches lacked the technology to accomplish two key activities that appear critical to the long-term success of treatments (1) none of these approaches included a systematic way to improve the effectiveness of their approach (i.e., self-learning), and (2) these approaches did not have a way to personalize or individualize feedback or treatment that is optimized to a specific case. The latter is especially important based on theories of engagement (Perski et al. 2017) and precision mental health services (Bickman et al. 2016).

\section{Reform 2.0: Using Artificial Intelligence and Precision Mental Health to Augment Current Innovative Approaches}

With the recent developments in AI, we are now able to develop software applications or platforms that can better identify which data to collect and feedback and to personalize treatment including making recommendations to support treatment delivery by clinicians. These applications are capable of personalizing the response and feedback and can be tailored to client needs and clinicians' delivery of the intervention. Moreover, it is now possible for these applications to improve with continuous learning.

To be clear, the promise of AI to improve mental health services and treatment outcomes at this point is just a promise. AI-based applications have the potential to greatly improve mental health services but at this point this should be interpreted as a strong hypothesis. As I point out (Bickman 2020) our work with AI has a long way to go. We are just at the beginning stage in utilizing AI to predict clinical outcomes, but we have not even scratched the surface on intervening with AI to improve outcomes.

\section{But it takes more than just Software and Technology to Implement Reform 2.0: The AI-Powered Platform and Support Services}

Several colleagues and I have developed a concept that at this point is still partially aspirational, but recognizes that the problems we face will not be solved by software or technology alone. We have learned the hard lessons over the last several decades about the importance of proper implementation in dealing with real-world services. Thus, we have developed a system that focuses on bringing together the relevant parties in implementing AIenhanced services. These include researchers, software vendors, clients, and providers of mental health services. It is described here as an example of the type of implementation support we need in Reform 2.0.

The Practice to Research Acceleration Center (PRAC) is an Implementation/ Acceleration/ Technology professional service that is part of the Feedback Research Institute (https://www.org/feedbackresearch.org). PRAC provides the human intelligence and experience necessary to effectively implement Reform 2.0. The central functions and activities of PRAC's approach include:

- Serving as a translator/conduit among provider agencies, researchers, and software vendors,

- Developing and maintaining a common database of client-level data that can be used for research, devel- 
opment, and dissemination. We especially focus on what might be called micro-data or data typically collected by measurement feedback systems (MFS's) that are longitudinal and collected contemporaneously with treatment and

- Providing an AI-enhanced software platform that is capable of improving service outcomes by intelligently identifying the appropriate data to collect and feedback

o This AI-powered platform is capable of self-learning and thus improving both the platform's and the vendors' treatments' performance over time.

PRAC will help community providers select and modify the technology, as well as support the training and implementation of that technology. Much of the current platform's development is through PRAC's Social Impact AI Lab (SIAIL), which is a consortium of nonprofit social services agencies and technology providers working together to develop AI applications to address the efficacy of interventions for youth and families receiving mental health and social services. The Lab developed the nascent SIAIL Platform using AI to optimize software innovations and provide feedback to clinicians on multiple client risk factors and social determinants of health. SIAIL's AI heuristics are based on continuous improvement to optimize clinical effectiveness with targeted feedback on areas of imminent risk. The goal is to deliver personalized or individualized feedback and treatment that are uniquely appropriate for each case. The standardization of data and utilization of a common data model allows for cross-application development. Furthermore, this common platform will make available to vendors the latest AI approaches to improve their products that individually they could probably not afford to employ.

\section{A Special Emphasis on Under-Resourced Communities}

PRAC's model places particular importance on underresourced communities. Communities that need modern applications are often least likely to be able to afford them, and least prepared to implement the latest technologies (Eberhardt et al. 2020). Moreoeover, there is a plethora of research that indicates that underserved communities suffer from the worst health outcomes (Butler and Rodgers 2019). In recognition of these problems, PRAC is working to provide the latest technologies, services, and resources at little or no cost to small nonprofits and community mental/ behavioral health providers. The goal is to facilitate linkages between providers in under-resouced communities to use SIAIL, guide vendor partnerships with software developers and provide technical assistance and resources to communities so they become the beneficiaries of the latest technological innovations and applications. Additionally, PRAC will guide community providers and prepare them and their organizations to adopt, adapt, and implement technologies to fit their needs. While mental health and social services communities have been "siloed" in the past, both of these services could benefit from an integrative approach that leverages technology, innovation, and implementation support. In Reform 2.0 we need to strive to rethink innovations in the area of mental health and social services and develop novel intervention approaches using the latest technology such as AI for the benefit of all.

\section{References}

American Psychological Association, Society of Clinical Psychology (Division 12), (2004), A Guide to Beneficial Psychotherapy, www. apa.org/divisions/div12/rev_est/index.html

Bickman, L. (2020). Improving Mental Health Services: A 50-Year Journey from Randomized Experiments to Artificial Intelligence and Precision Mental Health. Administration and Policy In Mental Health. https://doi.org/10.1007/s10488-020-01065-8.

Bickman, L., Lyons, A., \& Wolpert, M. (2016). Achieving Precision Mental Health through Effective Assessment, Monitoring, and Feedback Processes. Administration and Policy in Mental Health and Mental Health Ser-vices Research. https://doi.org/10.1007/ s10488-016-0718-5.

Bickman, L. (2008). A measurement feedback system (MFS) is necessary to improve mental health outcomes. Journal of the American Association of Child and Adolescent Psychiatry, 47, 1114-1119. https://doi.org/10.1097/CHI.0b013e3181825af8.

Bickman, L., Smith, C. M., Lambert, E. W., \& Andrade, A. R. (2003). Evaluation of a congressionally mandated wraparound demonstration Featured article. Journal of Child and Family Studies, 12(2), 135-156.

Bickman, L., \& Noser, K. (1999). Meeting the challenges in the delivery of child and adolescent mental health services in the next millennium: The continuous quality improvement approach. Applied and Preventive Psychology, 8, 247-255. https://doi.org/10.1016/ S0962-1849(05)80039-3.

Bickman, L., Karver, M., \& Schut, L. J. A. (1997). Clinician reliability and accuracy in judging appropriate level of care. Journal of Consulting and Clinical Psychology, 65(3), 515-520. https://doi. org/10.1037/0022-006X.65.3.515.

Burns, B. J., \& Friedman, R. M. (1990). Examining the research base for child mental health services and policy. The Journal of Mental Health Administration, 17, 87-98. https://doi.org/10.1007/BF025 18583.

Butler, A. M., \& Rodgers, C. (2019). Developing a Policy Brief on Child Mental Health Disparities to Promote Strategies for Advancing Equity among Racial/Ethnic Minority Youth. Ethnicity and Disease, 29(Suppl 2), 421-426. https://doi.org/10.18865 led.29.S2.421.

Chorpita, B. F., Yim, L. M., Donkervoet, J. C., Arensdorf, A., Amundsen, M. J., McGee, C., et al. (2002). Toward large-scale implementation of empirically supported treatments for children: A review and observations by the Hawaii empirical basis to services task force. Clinical Psychology: Science and Practice, 9, 165-190. https://doi.org/10.1111/j.1468-2850.2002.tb00504.x.

Chowanec, G. D. (1994). Continuous quality improvement: conceptual foundations and application to mental health care. Hospital 
and Community Psychiatry, 45, 789-793. https://doi.org/10.1176/ ps.45.8.789.

Eberhardt, P., Wial. H., \& Yee, D., (2020) The New Face of UnderResourced Communities, Boston:Initiative for a Competitive Inner City. https://icic.org/wp-content/uploads/2020/10/The-New-Faceof_Under-Resourced-Communities.pdf

Hedlund, J. L., Vieweg, M. S., \& Chow, D. W. (1985). Mental Health Computing in the 1980s. Computers in Human Services, 1(2), 1-31. https://doi.org/10.1300/J407v01n02_01.

Lambert, M. J., Whipple, J. L., Hawkins, E. J., Vermeersch, D. A., Nielsen, S. L., \& Smart, D. W. (2003). Is it time for clinicians to routinely track patient outcome? A meta-analysis. Clin Psychol Sci Pract, 10(3), 288-301. https://doi.org/10.1093/clipsy.bpg025.

Linardon, J., \& Fuller-Tyszkiewicz, M. (2020). Attrition and adherence in smartphone-delivered interventions for mental health problems: A systematic and meta-analytic review. Journal of Consulting and Clinical Psychology, 88(1), 1-13. https://doi.org/10.1037/ccp00 00459.

Lyon, A. R., Dopp, A. R., Brewer, S. K., et al. (2020). Designing the Future of Children's Mental Health Services. Administration and Policy In Mental Health, 47, 735-751. https://doi.org/10.1007/ s10488-020-01038-x.
Perski, O., Blandford, A., West, R., \& Michie, S. (2017). Conceptualising engagement with digital behaviour change interventions: a systematic review using principles from critical interpretive synthesis. Transl Behav Med., 7(2), 254-267. https://doi.org/10.1007/ s13142-016-0453-1.

Strecher, V. (2007). Internet methods for delivering behavioral and health-related interventions (eHealth). Annu Rev Clin Psychol., 3, 53-76. https://doi.org/10.1146/annurev.clinpsy.3.022806.091428.

Weisz, J. R., Kuppens, S., Ng, M. Y., Vaughn-Coaxum, R. A., Ugueto, A. M., Eckshtain, D., et al. (2019). Are psychotherapies for young people growing stronger? Tracking trends over time for youth anxiety, depression, attention-deficit/hyperactivity disorder, and conduct problems. Perspectives on Psychological Science, 14(2), 216-237.

Publisher's Note Springer Nature remains neutral with regard to jurisdictional claims in published maps and institutional affiliations. 